\title{
Comparison between Vertical Handoff Decision Algorithms for Heterogeneous Wireless Networks
}

\author{
Enrique Stevens-Navarro and Vincent W.S. Wong \\ Department of Electrical and Computer Engineering \\ The University of British Columbia, Vancouver, Canada \\ e-mail: $\{$ enriques, vincentw\} @ece.ubc.ca
}

\begin{abstract}
The next generation wireless networks will support the vertical handoff mechanism in which users can maintain the connections when they switch from one network to another (e.g., from IEEE 802.11b to CDMA 1xRTT network, and vice versa). Although various vertical handoff decision algorithms have been proposed in the literature recently, there is a lack of performance comparisons between different schemes. In this paper, we compare the performance between four vertical handoff decision algorithms, namely, MEW (Multiplicative Exponent Weighting), SAW (Simple Additive Weighting), TOPSIS (Technique for Order Preference by Similarity to Ideal Solution), and GRA (Grey Relational Analysis). All four algorithms allow different attributes (e.g., bandwidth, delay, packet loss rate, cost) to be included for vertical handoff decision. Results show that MEW, SAW, and TOPSIS provide similar performance to all four traffic classes. GRA provides a slightly higher bandwidth and lower delay for interactive and background traffic classes.
\end{abstract}

\section{INTRODUCTION}

Currently, there are various wireless access networks deployed. Examples include wireless cellular networks, WLANs (Wireless Local Area Networks), and wireless PANs (Personal Area Networks). There is an emerging trend that some of the mobile devices are equipped with multiple network interface cards, which are capable of connecting to different wireless access networks. Users with multimedia-enabled wireless devices are expected to obtain both real-time services (e.g., voice, video conferencing), and non-real time services (e.g., Simple Message Service (SMS), Multimedia Message Service (MMS)).

The next generation wireless networks will provide a service which allows a user to launch multimedia Internet applications anywhere at any time from diverse networks over an IP (Internet Protocol) backbone. It is foreseeable that users may want to maintain the connections when they switch from one network to another (e.g., from IEEE 802.11b to CDMA 1xRTT network, and vice versa). This is known as vertical handoff. Vertical handoff is different from conventional horizontal handoff where the mobile devices move from one base station to another within the same network.

Within the 3GPP (Third Generation Partnership Project) and 3GPP2 standardization groups, there are proposals describing the interconnection requirements between 3GPP systems and WLANs [1], [2]. Within the IEEE, the 802.21 Media Independent Handover (MIH) Working Group is working towards a standard to facilitate vertical handoff between IEEE 802 technologies and 3GPP/3GPP2 networks [3].
There are several challenging issues on vertical handoff support [4]. The vertical handoff decision may depend on the bandwidth available for each wireless access network, the ISP (Internet Service Provider) charge for the network connection, the power usage requirements, and the current battery status of the mobile device. The vertical handoff operation should provide authentication of the mobile users, incur a low control overhead, and maintain the connections such that packet loss and transfer delay are minimized.

In general, the vertical handoff process can be divided into three main steps [5], [6], namely system discovery, handoff decision, and handoff execution. During the system discovery phase, mobile terminals equipped with multiple interfaces have to determine which networks can be used and the services available in each network. The networks may also advertise the supported data rates for different services. During the handoff decision phase, the mobile device determines which network it should connect to. The decision may depend on various parameters including the available bandwidth, delay, jitter, access cost, transmit power, current battery status of the mobile device, and the user's preferences. During the handoff execution phase, connections need to be re-routed from the existing network to the new network in a seamless manner. This phase also includes the authentication and authorization, and the transfer of user's context information.

Although various vertical handoff decision algorithms have been proposed in the literature recently [7]-[10], there is a lack of performance comparisons between different schemes. In this paper, we compare the performance between four vertical handoff decision algorithms, namely, MEW (Multiplicative Exponent Weighting), SAW (Simple Additive Weighting) [9], TOPSIS (Technique for Order Preference by Similarity to Ideal Solution) [9], and GRA (Grey Relational Analysis) [10]. All four algorithms allow different attributes (e.g., bandwidth, delay, packet loss rate, cost) to be included for vertical handoff decision. Simulation results show that MEW, SAW, and TOPSIS provide similar performance to all four traffic classes. GRA provides a slightly higher bandwidth and lower delay for interactive and background traffic classes.

The rest of the paper is organized as follows. The background and related work on vertical handoff decision are given in Section II. The performance comparisons between different vertical handoff algorithms are presented in Section III. Conclusions and future work are presented in Section IV. 


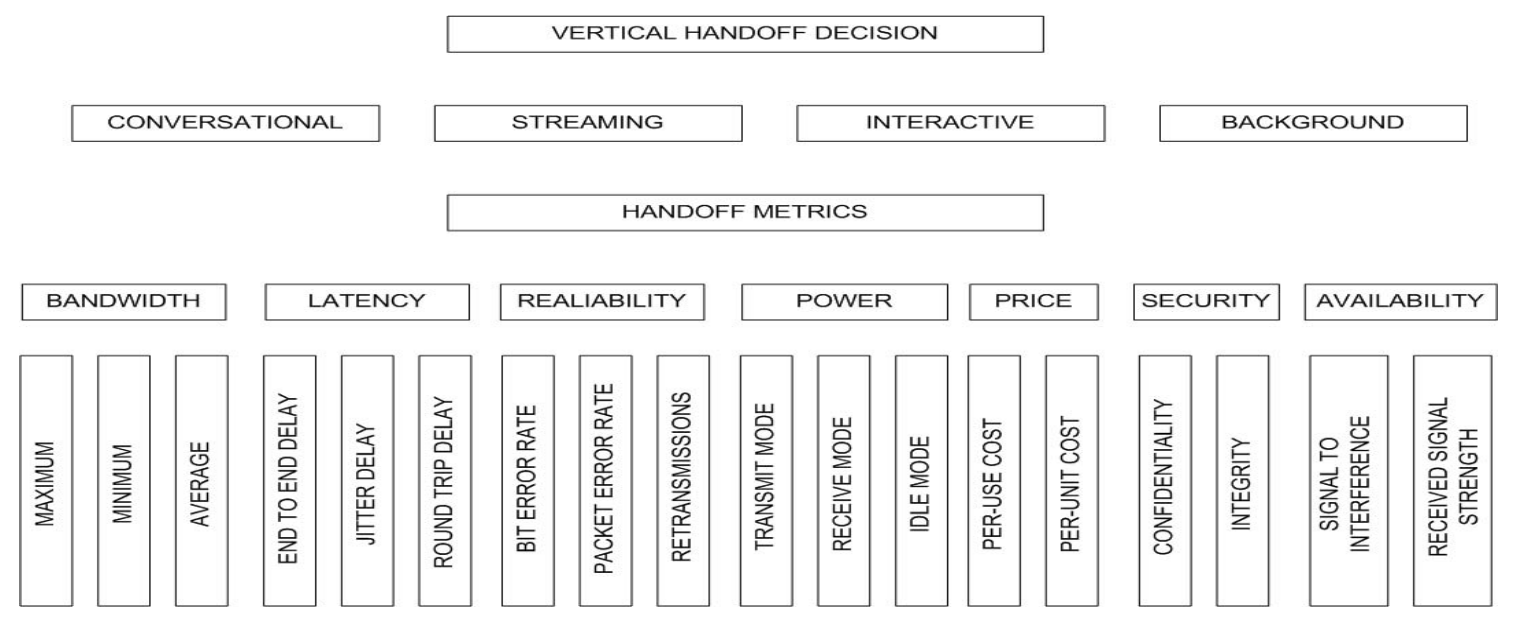

Fig. 1. Handoff metrics and traffic classes.

\section{BACKGROUND AND RELATED WORK}

Both QoS parameters and handoff metrics are required for vertical handoff decision [5]. The QoS parameters (e.g., bandwidth, delay bound) are specified by the applications. The information of different handoff metrics are gathered during the system discovery phase. Fig. 1 shows various handoff metrics and traffic classes (e.g., conversational, streaming, interactive, background). The handoff metrics and QoS parameters are categorized under different groups (e.g., bandwidth, latency, power, price, security, reliability, availability).

Various vertical handoff decision mechanisms have been proposed recently. In [7], the handoff decision mechanism is formulated as an optimization problem. Each candidate network is associated with a cost function. The decision is to select the network which has the lowest cost value. The cost function depends on a number of criteria, including the bandwidth, delay and power requirement. Appropriate weight factor is assigned to each criterion to account for its importance. In [8], an Active Application Oriented (AOO) vertical handoff decision mechanism is proposed. The decision mechanism considers the QoS parameters required for the applications (e.g., minimum and maximum bandwidth requirement for voice service). Each candidate network is associated with a utility function. The chosen network is the one which provides the highest utility value. The utilization function is a weighted sum of various normalized QoS parameters.

In the following sub-section, we discuss the vertical handoff decision algorithms which are being used in our performance comparisons.

\section{A. SAW and TOPSIS}

In [9], the vertical handoff decision is formulated as a fuzzy MADM (Multiple Attribute Decision Making) problem. Fuzzy logic is used to represent the imprecise information of some attributes and user preferences. For example, in fuzzy logic, the sojourn time can be represented as \{short, long, very long\}. The fuzzy MADM method consists of two steps. The first step is to convert the fuzzy data into a real number. The second step is to use classical MADM methods to determine the ranking order of the candidate networks. Two classical MADM methods are proposed: SAW (Simple Additive Weighting) and TOPSIS (Technique for Order Preference by Similarity to Ideal Solution).

In SAW, the overall score of a candidate network is determined by the weighted sum of all the attribute values. The score of each candidate network $i$ is obtained by adding the normalized contributions from each metric $r_{i j}$ multiplied by the importance weight assigned $w_{j}$ of metric $j$. The selected network $A_{S A W}^{*}$ is:

$$
A_{S A W}^{*}=\arg \max _{i \in M} \sum_{j=1}^{N} w_{j} r_{i j}
$$

where $N$ is the number of parameters, and $M$ denotes the number of candidate networks.

In TOPSIS, the selected candidate network is the one which is the closest to the ideal solution (and the farthest from the worst case solution). The ideal solution is obtained by using the best values for each metric. Let $c_{i}^{*}$ denote the relative closeness (or similarity) of the candidate network $i$ to the ideal solution. The selected network $A_{T O P}^{*}$ is:

$$
A_{T O P}^{*}=\arg \max _{i \in M} c_{i}^{*} .
$$

\section{B. GRA}

In [10], the network selected is based on Analytic Hierarchy Process (AHP) and Grey Relational Analysis (GRA). AHP decomposes the network selection problem into several subproblems and assigns a weight value for each sub-problem. GRA is then used to rank the candidate networks and selects the one with the highest ranking.

The ranking of GRA is performed by building grey relationships with a positive ideal network. A normalization process to deal with benefit and cost metrics is required and the Grey Relational Coefficient (GRC) of each network is calculated. The GRC is the score used to describe the similarity between each candidate network and the ideal network. The selected network is the one which has highest similarity to the ideal network. The selected network $A_{G R A}^{*}$ is: 


$$
A_{G R A}^{*}=\arg \max _{i \in M} \Gamma_{0, i}
$$

where $\Gamma_{0, i}$ is the GRC of network $i$.

\section{C. $M E W$}

The Multiplicative Exponent Weighting (MEW) is another MADM scoring method [11]. The vertical handoff decision problem can be expressed as a matrix form, where each row $i$ corresponds to the candidate network $i$ and each column $j$ corresponds to an attribute (e.g., bandwidth, delay).

The score $S_{i}$ of network $i$ is determined by the weighted product of the attributes (or metrics):

$$
S_{i}=\prod_{j=1}^{N} x_{i j}^{w_{j}},
$$

where $x_{i j}$ denotes attribute $j$ of candidate network $i, w_{j}$ denotes the weight of attributed $j$, and $\sum_{j=1}^{N} w_{j}=1$.

Note that in eqn. (4), $w_{j}$ is a positive power for benefit metrics $x_{i j}^{w_{j}}$, and a negative power for cost metrics $x_{i j}^{-w_{j}}$. Since the score of a network obtained by MEW does not have an upper bound [11], it is convenient to compare each network with the score of the positive ideal network $A^{* *}$.

This network is defined as the network with the best values in each metric. For a benefit metric, the best value is the largest. For a cost metric, the best value is the lowest.

The value ratio $R_{i}$ between network $i$ and the positive ideal is calculated by:

$$
R_{i}=\frac{\prod_{j=1}^{N} x_{i j}^{w_{j}}}{\prod_{j=1}^{N}\left(x_{i j}^{* *}\right)^{w_{j}}}
$$

where $0 \leq R_{i} \leq 1$.

The selected network $A_{M E W}^{*}$ is:

$$
A_{M E W}^{*}=\arg \max _{i \in M} R_{i}
$$

The importance weights $w_{j}$ required for MEW and other vertical handoff decision algorithms are calculated by using the eigenvector method. The weight values depend on the QoS requirements of the traffic classes.

\section{Method to determine the Weights}

The vertical handoff decision algorithms which we consider for performance comparisons (i.e., MEW, SAW, TOPSIS and GRA) require information about the relative importance of each metric or attribute. It is usually given by a set of weights $w_{j}$. The eigenvector method used by the AHP can be used to determine the weights [12]. It requires answers to a sequence of comparisons between a pair metrics. The answers are given by using the fundamental 1-9 AHP scale [13].

If $B$ is defined as an AHP comparison matrix, then by solving the system $B w=n_{\max } w$ (where $n_{\max }$ is the largest eigenvalue of $B$ ), the priority or importance vector $w$ can be obtained. To verify the consistency in judgment of the comparisons in $B$, the Consistency Ratio (CR) is used as an indicator. In practice, a $\mathrm{CR} \leq 0.1$ is considered to be acceptable [13].

\section{Performance Comparison}

In this section, we present results for the simulation based performance comparisons between four different vertical handoff decision algorithms, namely: SAW [9], TOPSIS [9], GRA [10], and MEW. The coverage area is assumed to be collocated with four networks: UMTS, GPRS, and two WLANs.

Four traffic classes defined by 3GPP in [14] are considered: conversational, streaming, interactive, and background. Each traffic class is associated with four different QoS parameters or attributes: available bandwidth, end-to-end delay, jitter, and bit error rate (BER). For the simulation, the connection lifetime of the mobile device is assumed to follow an exponential distribution. The average connection lifetime $1 / \mu$ is varied from 1 to 10 minutes. For each network $i$, the time between the attribute values being changed also follows an exponential distribution with the mean equals to $1 / \gamma_{i}$. We use $1 / \gamma_{1}=$ $1 / \gamma_{2}=4 \mathrm{~min}, 1 / \gamma_{3}=1 / \gamma_{4}=1 \mathrm{~min}$. The change in the value of the metrics evolve according to a Markov chain with the following state transition matrix:

$$
\left[\begin{array}{ccccc}
0.5 & 0.5 & 0 & 0 & 0 \\
0.25 & 0.5 & 0.25 & 0 & 0 \\
0 & 0.25 & 0.5 & 0.25 & 0 \\
0 & 0 & 0.25 & 0.5 & 0.25 \\
0 & 0 & 0 & 0.5 & 0.5
\end{array}\right] .
$$

For the bandwidth vector, we have: network 1 (UMTS) with $[32,64,128,256,512,1024,2048] \mathrm{kbps}$; network 2 (GPRS) with $[21,42,64,85,107,128,149,171] \mathrm{kbps}$; and networks 3 and 4 (WLAN) with $[1,2,5.5,11]$ Mbps. For the delay vector, we have: network 1 with $[190,160,130,100,70,40,10] \mathrm{ms}$; network 2 with $[185,160,135,110,85,60,35,10] \mathrm{ms}$; and networks 3 and 4 with $[160,110,60,10] \mathrm{ms}$. The four networks use the same vectors for jitter and BER. The values for the jitter vector are: $[3,5,7,9,11]$ msecs; the values for the BER vector are $\left[10^{-2}, 10^{-3}, 10^{-4}, 10^{-5}, 10^{-6}\right]$. Each element of a vector represents the value of the attribute in the state of the chain and the matrix provides the transition probability.

The four traffic classes have different QoS requirements [14]. To account for this fact, we assigned different weights for the same attribute between different traffic classes. The AHP matrices for the four traffic classes are shown in Table I. The weights determined by using the eigenvector method are shown in Table II.

\section{A. Results}

Fig. 2 shows the average bandwidth allocated to each traffic class under different vertical handoff decision algorithms. The mean value is obtained by averaging the values from 1000 connections. Results from Fig. 2 (a) show that the conversational traffic class receives approximately the same amount of bandwidth when any one of the four handoff decision algorithms is being used. This can also be observed for the streaming traffic class (see Fig. 2 (b)). The interactive 
TABLE I

AHP MATRICES FOR EACH TRAFFIC CLASS.

\begin{tabular}{|l||c|c|c|c|}
\hline Conversational & BER & Delay & Jitter & Bandwidth \\
\hline \hline BER & 1 & $1 / 9$ & $1 / 9$ & 1 \\
Delay & 9 & 1 & 1 & 9 \\
Jitter & 9 & 1 & 1 & 9 \\
Bandwidth & 1 & $1 / 9$ & $1 / 9$ & 1 \\
\hline \hline Streaming & BER & Delay & Jitter & Bandwidth \\
\hline \hline BER & 1 & $1 / 5$ & $1 / 9$ & $1 / 9$ \\
Delay & 5 & 1 & $1 / 5$ & $1 / 5$ \\
Jitter & 9 & 5 & 1 & 1 \\
Bandwidth & 9 & 5 & 1 & 1 \\
\hline \hline Interactive & BER & Delay & Jitter & Bandwidth \\
\hline \hline BER & 1 & 5 & 9 & 5 \\
Delay & $1 / 5$ & 1 & 5 & 1 \\
Jitter & $1 / 9$ & $1 / 5$ & 1 & $1 / 5$ \\
Bandwidth & $1 / 5$ & 1 & 5 & 1 \\
\hline \hline Background & BER & Delay & Jitter & Bandwidth \\
\hline \hline BER & 1 & 9 & 9 & 5 \\
Delay & $1 / 9$ & 1 & 1 & $1 / 5$ \\
Jitter & $1 / 9$ & 1 & 1 & $1 / 5$ \\
Bandwidth & $1 / 5$ & 5 & 5 & 1 \\
\hline
\end{tabular}

TABLE II

IMPORTANCE WEIGHTS PER CLASS AND CONSISTENCY RATIO (CR).

\begin{tabular}{|l|c|c|c|c|c|}
\hline Traffic Class & BER & Delay & Jitter & Bandwidth & CR \\
\hline Conversational & 0.04998 & 0.45002 & 0.45002 & 0.04998 & 0.000 \\
Streaming & 0.03737 & 0.11380 & 0.42441 & 0.42441 & 0.049 \\
Interactive & 0.63593 & 0.16051 & 0.04304 & 0.16051 & 0.049 \\
Background & 0.66932 & 0.05546 & 0.05546 & 0.21976 & 0.049 \\
\hline
\end{tabular}

and background classes receive slightly more bandwidth when GRA is used (see Figs. 2 (c) and (d)).

Fig. 3 shows the average delay of each traffic class under different vertical handoff decision algorithms. Results show that the delay performance for GRA, SAW, TOPSIS, and MEW are very close for conversational and streaming traffic classes. The interactive and background classes have a slightly smaller delay when GRA is used.

\section{B. Sensitivity Analysis}

In this section, we investigate the sensitivity of the assigned weights to the network selection. For conversational and streaming traffic classes, the weight of the jitter is varied from 0 to 1 . The weights for other metrics are varied in proportion to the values specified in Table II. For interactive and background traffic classes, the weight of BER is varied accordingly. The values of other metrics in each network are stated in Table III.

Figs. 4 (a) and (b) show that when the weight of the jitter increases, eventually all four algorithms select network 1 which has the lowest jitter. Figs. 4 (c) and (d) show that when

TABLE III

VALUES FOR SENSITIVITY ANALYSIS

\begin{tabular}{|c|c|c|c|c|}
\hline No. & Bandwidth (Kbps) & Delay (ms) & Jitter (ms) & BER \\
\hline 1 & 1000 & 15 & 5 & $10^{-4}$ \\
2 & 300 & 10 & 10 & $10^{-4}$ \\
3 & 25000 & 25 & 15 & $10^{-5}$ \\
4 & 11000 & 35 & 10 & $10^{-6}$ \\
\hline
\end{tabular}
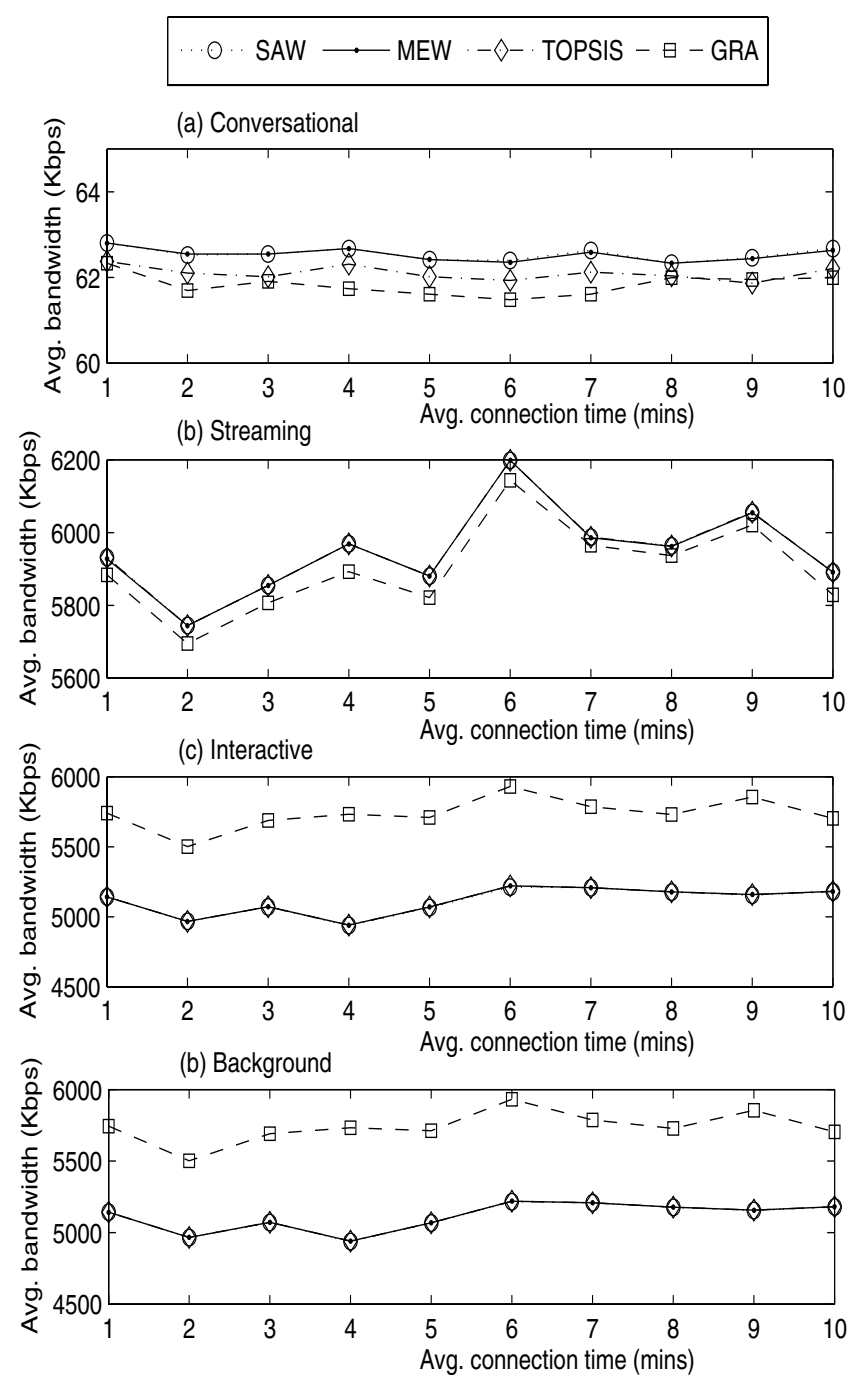

Fig. 2. Average bandwidth for the four traffic classes.

the weight of BER increases, eventually all four algorithms select network 4 which has the lowest BER value.

Table IV shows the percentage of time that all algorithms select the same network. MEW, SAW and TOPSIS select the same network between $90 \%-99 \%$ of time. The four algorithms select the same network between $72 \%-87 \%$ of time.

\section{CONCLUSiOnS}

In this paper, we presented the results for the performance comparison between four different vertical handoff decision algorithms, namely: namely, MEW (Multiplicative Exponent Weighting), SAW (Simple Additive Weighting) [9], TOPSIS (Technique for Order Preference by Similarity to Ideal Solution) [9], and GRA (Grey Relational Analysis) [10]. The attributes that we considered in the simulation model included bandwidth, delay, jitter, and BER. Results show that MEW, SAW, and TOPSIS provide similar performance to all four traffic classes. GRA provides a slightly higher bandwidth and lower delay for interactive and background traffic classes. Results also showed that all four algorithms depend on the 
○. SAW — MEW - $-\odot-$ TOPSIS $-\boxminus-$ GRA

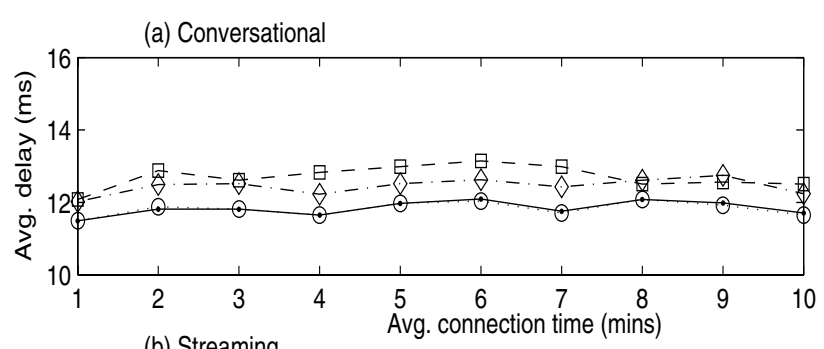

(b) Streaming

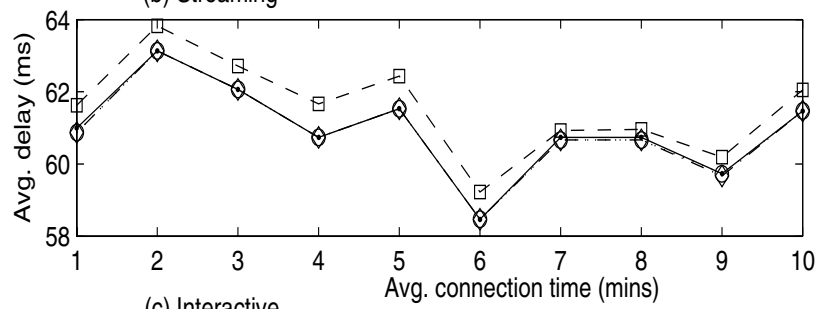

(c) Interactive

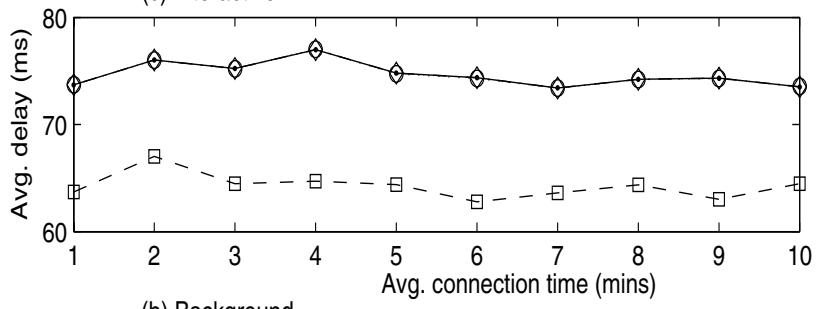

(b) Background

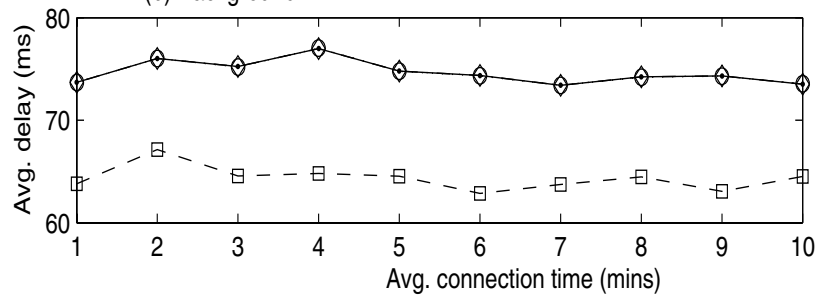

Fig. 3. Average delay for the four traffic classes

importance weights assigned to the parameters. For future work, we plan to investigate other techniques (besides AHP) for the determination of the weight values. We will also consider the vertical handoff execution procedures.

\section{ACKNOWLEDGMENT}

This work was supported by Programa de Mejoramiento del Profesorado (PROMEP) from Mexico and Bell Canada.

\section{REFERENCES}

[1] 3GPP, "Requirements on 3GPP System to Wireless Local Area Network (WLAN) Interworking," TS 22.234 (v7.0.0), January 2005.

[2] 3GPP2, "3GPP2-WLAN Interworking," S.R0087-0 (v1.0), July 2004.

[3] IEEE, "Draft IEEE Standard for Local and Metropolitan Area Networks: Media Independent Handover Services," P802.21/D00.01, July 2005.

[4] I. Akyildiz, J. Xie, and S. Mohanty, "A Survey of Mobility Management in Next-Generation All-IP-Based Wireless Systems," IEEE Wireless Communications, vol. 11, no. 4, August 2004.

[5] J. McNair and F. Zhu, "Vertical Handoffs in Fourth-generation Multinetwork Environments," IEEE Wireless Comm., vol. 11, no. 3, June 2004

[6] W. Chen, J. Liu, and H. Huang, "An Adaptive Scheme for Vertical Handoff in Wireless Overlay Networks," in Proc. of ICPADS'04, Newport Beach, CA, July 2004. (a) Conversational

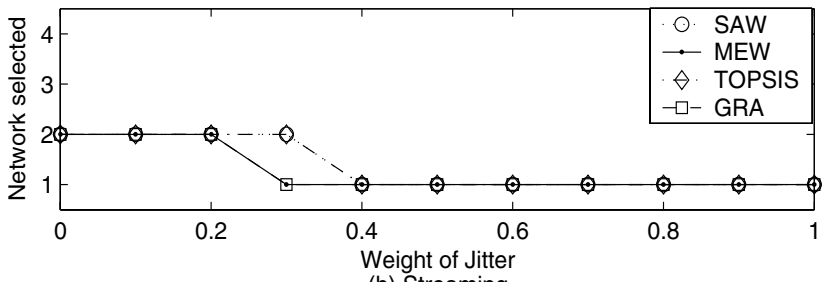

(b) Streaming

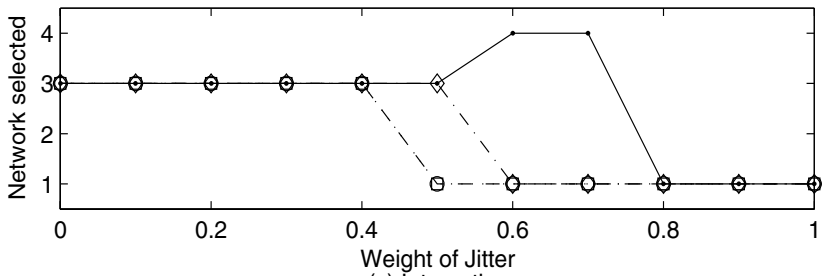

(c) Interactive

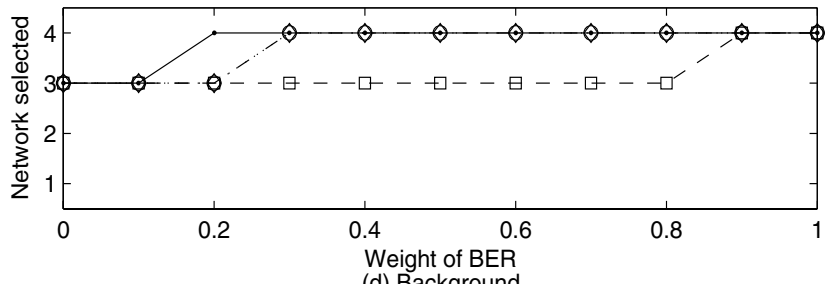

(d) Background

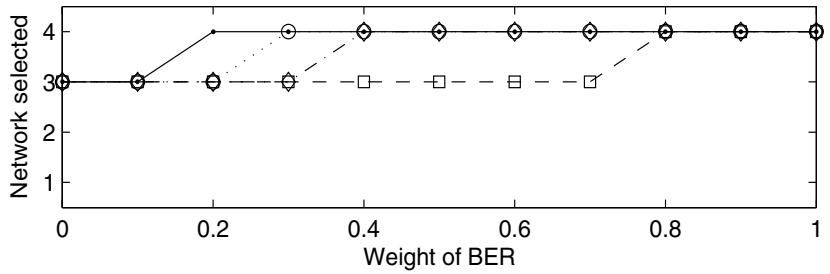

Fig. 4. Sensitivity Analysis.

TABLE IV

PERCENTAGE OF TIME THAT THE SAME NETWORK BEING SELECTED.

\begin{tabular}{|l|c|c|c|c|}
\hline Methods & $\begin{array}{c}\text { Conver- } \\
\text { sational }\end{array}$ & Streaming & Interactive & Background \\
\hline MEW / SAW & $92.36 \%$ & $96.47 \%$ & $98.44 \%$ & $98.84 \%$ \\
MEW / TOP & $90.60 \%$ & $95.28 \%$ & $99.73 \%$ & $99.80 \%$ \\
MEW / GRA & $79.97 \%$ & $88.96 \%$ & $72.45 \%$ & $73.98 \%$ \\
SAW / TOP & $97.64 \%$ & $98.59 \%$ & $98.55 \%$ & $99.02 \%$ \\
SAW / GRA & $86.04 \%$ & $90.84 \%$ & $73.31 \%$ & $74.05 \%$ \\
TOP / GRA & $84.74 \%$ & $90.42 \%$ & $72.52 \%$ & $74.05 \%$ \\
All & $78.36 \%$ & $87.91 \%$ & $72.34 \%$ & $73.91 \%$ \\
\hline
\end{tabular}

[7] F. Zhu and J. MacNair, "Optimizations for Vertical Handoff Decision Algorithms," in Proc. IEEE WCNC'04, Atlanta, GA, March 2004.

[8] W. Chen and Y. Shu, "Active Application Oriented Vertical Handoff in Next Generation Wireless Networks," in Proc. IEEE WCNC'05, New Orleans, LA, March 2005.

[9] W. Zhang, "Handover Decision Using Fuzzy MADM in Heterogeneous Networks," in Proc. IEEE WCNC'04, Atlanta, GA, March 2004.

[10] Q. Song and A. Jamalipour, "A Network Selection Mechanism for Next Generation Networks," in Proc. IEEE ICC'05, Seoul, Korea, May 2005.

[11] K. Yoon and C. Hwang, Multiple Attribute Decision Making: An Introduction. Sage Publications, 1995.

[12] C. Hwang and K. Yoon, Multiple Attribute Decision Making: Methods and Applications. Springer-Verlag, 1981.

[13] T. Saaty, The Analytic Hierarchy Process: Planning, Priority Setting, Resource Allocation. Mc-Graw Hill, 1980.

[14] 3GPP, "QoS Concepts and Architecture," TS 22.107 (v6.3.0), June 2005. 